\title{
Early Identification of COVID-19 Using Dynamic Fuzzy Rule Based System
}

\author{
Mohammed Imran Basheer Ahmed, Atta-ur Rahman*, Mehwash Farooqui, Fatimah Alamoudi, Raghad Baageel, \\ Amjad Alqarni
}

Department of Computer Science, College of Computer Science and Information Technology, Imam Abdulrahman Bin Faisal University, P.O. Box 1982, Dammam 31441, Saudi Arabia

Corresponding Author Email: aaurrahman@iau.edu.sa

https://doi.org/10.18280/mmep.080517

Received: 22 May 2021

Accepted: 10 August 2021

\section{Keywords:}

COVID-19, early identification, fuzzy rule-based

system, dynamic membership function

\begin{abstract}
The undergoing research aims to address the problem of COVID-19 which has turned out to be a global pandemic. Despite developing some successful vaccines, the pace has not overcome so far. Several studies have been proposed in the literature in this regard, the present study is unique in terms of its dynamic nature to adapt the rules by reconfigurable fuzzy membership function. Based on patient's symptoms (fever, dry cough etc.) and history related to travelling, diseases/medications and interactions with confirmed patients, the proposed dynamic fuzzy rule-based system (FRBS) identifies the presence/absence of the disease. This can greatly help the healthcare professionals as well as laymen in terms of disease identification. The main motivation of this paper is to reduce the pressure on the health services due to frequent test assessment requests, in which patients can do the test anytime without the need to make reservations. The main findings are that there is a relationship between the disease and the symptoms in which some symptoms can indicate the probability of the presence of the disease such as high difficulty of breathing, cough, sore throat, and so many more. By knowing the common symptoms, we developed membership functions for these symptoms, and a model generated to distinguish between infected and non-infected people with the help of survey data collected. The model gave an accuracy of $88.78 \%$, precision of $72.22 \%$, sensitivity of $68.42 \%$, specificity of $93.67 \%$, and an f1-score of $69.28 \%$.
\end{abstract}

\section{INTRODUCTION}

In late December 2019, Wuhan, Hubei Province, China, suffered an outbreak of an undetermined disease known as pneumonia of unknown cause. The epidemic has infected 9,720 people in China, causing in 213 deaths, as well as 106 people in 19 other countries. A number of research laboratories reported a novel coronavirus $(\mathrm{nCoV})$ as the causative agent of this mysterious pneumonia a few days later. The World Health Organization has temporarily designated the causative virus as extreme acute respiratory syndrome coronavirus 2 (SARSCoV2) and the related infected disease as coronavirus disease 2019 (COVID19) [1]. Pneumonia was the first clinical sign of the SARSCoV2 associated disease COVID19 that allowed case detection. More recent studies, particularly among young children, describe gastrointestinal symptoms and asymptomatic infections [2]. COVID-19 symptoms will appear within 2 to 14 days after infection. Furthermore, the diseases can persist after 27 days in some cases. The average incubation period, according to Chinese researchers, is 5.2 days. Early deaths have been observed in older people, which is likely due to a lack of immunity, which promotes COVID-19 progression. As a result, strengthening our immune system is important. It is essential to recommend that people take supplements to help their immune systems [3]. Due to the high impact COVID-19 has made, a fuzzy inference system has been developed using MATLAB with the help of survey data that was published in Saudi Arabia and filled by people who have taken the COVID-19 test whether the result was positive or negative.

COVID-19 pandemic is not only a life threating disease, but it has also been greatly impacting almost every sector of human life around the globe. Like education, economics, transportation, businesses, education are few among these areas [4]. To further elaborate, the remainder of this work is organized as followed, section 2 contains a review of related literatures. Then section 3 contains the proposed methodology, and the survey data description, followed by section 4 which contains results and discussion. Finally, section 5 presents a comparison of our study and another similar study then section 6 contains the conclusion, future work, and recommendation emanating from this work.

\section{REVIEW OF RELATED LITERATURES}

Dhiman and Sharma [5] proposed a fuzzy inference system to diagnose COVID-19 disease using six input factors: ethanol, atmospheric temperature (AT), body temperature (BT), breath shortness (BS), cough, and cold, with the output factor divided into three linguistic categories denoting the severity level of the infected patients. They discovered that if the atmospheric temperature is moderate, there is a higher consumption of ethanol, and a somewhat higher body temperature, the infected patient would have a normal severity level. If the atmospheric temperature is low, the body temperature is medium, the amount of ethanol consumed is high, and the patient is coughing, the intensity level in the patient can be determined. 
Also, they discovered that when a patient has breathing difficulties or a sneezing problem when consuming a small amount of ethanol, the severity level is significantly higher than in other situations. As a result, their proposed inference method may aid in the accurate diagnosis of COVID-19infected patients.

Fatima et al. [6] proposed research uses an Internet of Things (IoT) approach allowed by Fuzzy Inference System (FIS) to predict the coronavirus. The system smartly monitors and predicts whether a person is infected with Coronavirus intelligently and efficiently. The statistical values used to forecast the use of FIS for coronavirus are the uncertain input function. The coronavirus diagnosis of five different forms of fuzzy variables is discussed in the article: flu, headache, cough, sore throat, and fever. The proposed system uses MATLAB 2019a for modeling, and it shows promising results in simulations. Five data sources and one performance factor are used to produce the reproduction results. In this paper, the proposed Fuzzy-based method is used to demonstrate a variety of outputs. The effects of the proposed system are very appealing.

In [7], a rule-based fuzzy inference system was developed in MATLAB. The system has 9 inputs which are fever, sore throat, travel history, age, diabetes, chest pain, breathing problem, cough, and loss of smell and taste, and one output. The linguistic variables for each input were as the following, fever as low, moderate and high, sore throat as low and high, travel history as not infected, doubt infection, and infected, age as less than 40 , greater than or equal to 40 and less than or equal to 50, and greater than or equal to 50, diabetes as normal (140 mg/dl), medium (170 mg/dl), and high (199 mg/dl), chest pain as low, medium and high, breathing problem as low, medium and high, cough as low, medium and high, and finally loss of smell and taste as low, medium and high. The system uses a triangular membership function and has 17 IF-THEN rules. The finding in this paper states that if the person was less than 40 and the fever was low, then the probability of being COVID-19 infected is negative. On the other hand, if the person was greater than 50 , has traveled to an infected country and has breathing problems then the probability of being COVID-19 is positive.

Khan et al. [8] proposed an IoMT-based smart monitoring fuzzy inference system for COVID-19 diagnosis. The system consists of two phases which are the training phase and the validation phase that communicate through the cloud. The training phase has three layers which are the sensory layer that consists of input parameters such as cough, CT-chest, family history, and fever, preprocessing layer in which missing values were handled, and the values were normalized, and the third layer which is the application layer. The application layer has two sublayers which are the prediction and the performance layer. The prediction layer is responsible for predicting the output while the performance layer in which the predicted result will be evaluated to measure the system performance. In the last phase, which is the validation phase, the trained system is used for prediction to validate whether the person is COVID-19 infected or not. Overall, the system has an accuracy of $83 \%$ and a precision of $82.5 \%$.

Shaban et al. [9] proposed a new Hybrid Diagnose Strategy (HDS) to detect patients with COVID-19 infection. A fuzzy inference engine and Deep Neural Network classifiers are used in the hybrid model. The dataset used in this paper is 279 medical records of patient's data. The records are classified into two categories, COVID-19 patients and non-COVID-19 patients. The attributes are gender, age, leukocytes (WBC), platelets, C- reactive protein (CRP), transaminases (AST), transaminases (ALT), gamma glutamil transferase (GGT), lactate dehydrogenase (LDH), neutrophils, lymphocytes, monocytes, eosinophils, and basophils. Twelve features are used in training and testing datasets. This strategy has the best performance as of the average value of precision, accuracy, Fmeasure, and recall compared to recent techniques. It provides 96.55\% precision, $97.658 \%$ accuracy, $96.615 \%$ F-measure, and $96.55 \%$ recall. Moreover, it has a $2.342 \%$ value of error which is considered the lowest value of error.

Shatnawi et al. [10] proposed a COVID-19 diagnosis system using an intelligent fuzzy inference system. Based on COVID-19 symptoms, it decides the risk level of COVID-19 infection. The Mamdani fuzzy model is applied to build the system. Nine symptoms were defined as the input variables to the system. It divides into two groups, the most common symptoms group, which are fever, dry cough, and tiredness, and the less common symptoms groups, which are headache, diarrhea, sore throat, loss of taste or smell, breathing difficulties, and conjunctivitis. For each symptom, two Gaussian membership functions were designed. All the input variable has a level range between 0 and 5 except the body temperature. It represents the fever variable, ranging from 36.5 to $42 \mathrm{C}$. the system defined thirteen fuzzy linguistic rules. The system produced the output using the "min" fuzzy operator and decide the percentage of the level of risk using the Centroid method. The proposed system's results were very desirable.

Zagrouba et al. [11], presented a simulation and modeling technique to predict the COVID-19 pandemic based on a supervised machine learning approach. In this regard, they have proposed a predictive framework incorporating Support Vector Machines (SVM) in the forecasting of a potential outbreak of COVID-19. The technique was promising in terms of accuracy and a $96.79 \%$ accuracy was observed in testing phase. A similar approach was proposed by Rahman et al. [12], where a multiple cross-fold validation. The proposed model was coined as CSDC-SVM (cloud-based smart detection algorithm using SVM). It was claim that the proposed model exhibited an accuracy of $98.4 \%$ with 15 -fold cross-validation.

Fuzzy system has been significantly proving their strength in classification, prediction and identification problems in various fields other than COVID-19 in the healthcare for disease prediction $[13,14]$. Like in telecommunications for better link adaptation [15-17], text and data mining for sake of automated text categorization and classification [18-20].

\section{MATERIAL \& METHODS}

\subsection{Description of the dataset}

The dataset was collected via a survey published to the public who had taken the COVID-19 test with a positive or negative result in Saudi Arabia. The dataset has nine categorical attributes and one numerical attribute. The attributes are gender, age, diseases, contact with a confirmed COVID-19 case, contact with a traveled person, traveled, fever range, cough range, breathing difficulty range, and sore throat pain rang. The dataset contains 104 samples, and two main classes, diagnosed with COVID-19 class contains 21 samples and not diagnosed with COVID-19 class contains 83 samples where it represents $79.8 \%$ of samples. Figure 1 represents the number of samples for each class. $69.2 \%$ of the samples are 
placed in the 30 or less age and the female category. $81.7 \%$ of the samples are placed in the normal fever range (35.6-37.5) category.

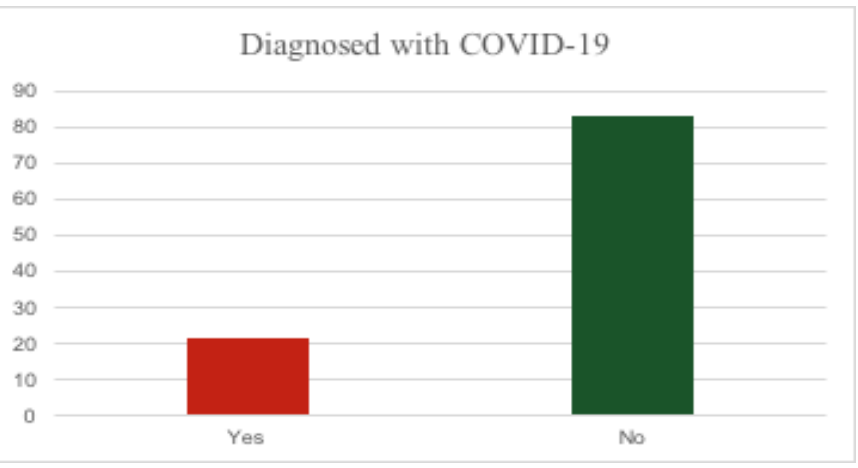

Figure 1. Number of samples per class category in the dataset

\subsection{Methodology}

\subsubsection{Rule-base fuzzy inference system}

A fuzzy inference system (FIS) is a system that uses fuzzy set theory to map inputs to outputs. Mamdani fuzzy inference system is used to develop the model along with the following parameters, centroid for the defuzzifer, max aggregation, minimum implication, minimum and method, and maximum or method. The combination of linguistic statements represents the fuzzy rules which categorize the way that FIS takes the decision to control the output or classify the inputs. The fuzzy rules are written in if-then form. Then, using the input membership functions, the inputs are fuzzified and combined based on the rules to set up a rule strength. They are then combining the rule strength and membership function output to find the outcomes of the rule. The outcomes are combined to obtain an output distribution, and finally, this output distribution is defuzzified [10]. The Figure 2 demonstrates the Mamdani fuzzy inference system.

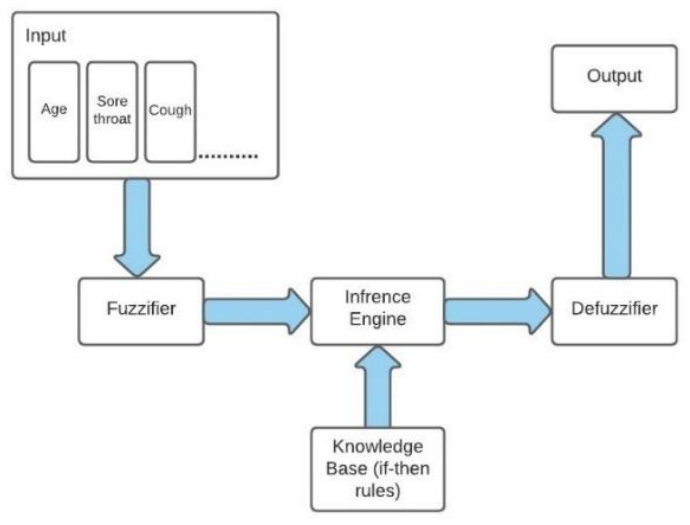

Figure 2. Mamdani rule-base fuzzy inference system

\subsubsection{Fuzzy logic}

Fuzzy logic adds an essential skill to bivalent logic, a capacity to reason precisely with imperfect knowledge which is imprecise, ambiguous, incomplete, inaccurate, unclear, or partially accurate in one or more respects. In fuzzy logic, reasoning results are supposed to be proving true, or in short, partially valid. Extended fuzzy logic adds an equally significant capacity to reason imprecisely with incomplete evidence. When precise reasoning is infeasible, unnecessarily expensive, or unneeded, this skill comes into play. P-validity of outcomes is desirable in extended fuzzy logic, but not necessary. A mode of reasoning that is fuzzily true is what is admissible, or f-valid for short. There are three principal rationales for the use of extended fuzzy logic. First, when a pvalid solution is infeasible. Second, when a p-valid solution carries an excessively high cost; and third, when there is no need for a p-valid solution, that is, when an f-valid solution is good enough. In much of everyday human reasoning, it is the third rationale that is preponderant [21].

Fuzzy logic can be used when the problem requires a complicated mathematical model and when the system behavior can be described in linguistic form. That is why fuzzy logic is considered a powerful tool for addressing uncertainties such as human thinking and reasoning. In addition, what should be underscored is that in dealing with many real-world problems, an f-valid solution based on a realistic model may be more useful than a p-valid solution based on an unrealistic model. Fuzzy logic has several advantages to be used like the flexibility, the fuzzy logic system can be modified just by adding or deleting rules. In addition to the simple mathematical concept [22].

\subsubsection{Membership function}

In the overall performance of fuzzy representation, membership functions (MFs) play a critical role. The MFs are the building blocks of the theory of the fuzzy set. Therefore, for a specific problem, the shapes of MFs are important since they affect a fuzzy inference system. They may have various forms, such as triangular, trapezoidal, Gaussian, etc. The only condition that an MF really must fulfill is that it must range from 0 to 1 . The fuzzy membership function enabled us to overcome the difficulty of having different control for a small change. This can be achieved without having to increase the number of control statements (rules). Membership function quantifies the degree of belonging of a variable in the fuzzy set to set itself. There are two main membership functions which are linear and curved (non-linear). Most applications use the linear type as it needs less computational time. As long as it maps the given data with a desirable degree of membership, the MFs may be of any shape and type. It is up to us to decide as far as the option of MFs is concerned. This is where individual degrees of freedom are given by the fuzzy method. With practice, one can come to understand which MF shape is better for the application under consideration [23, 24].

\subsubsection{Fuzzy inference system membership function}

Trapezoidal membership functions are designed for the most common COVID-19 symptoms and other factors that can impact the severity of the disease, such as age and chronic diseases. Reason behind considering the trapezoidal function over triangular function is its flexibility in handling. As there are four edges while in triangular function, there are three edges as investigated in Refs. [25, 26].

Table 1 displays the membership functions along with their ranges. 
Table 1. Membership functions

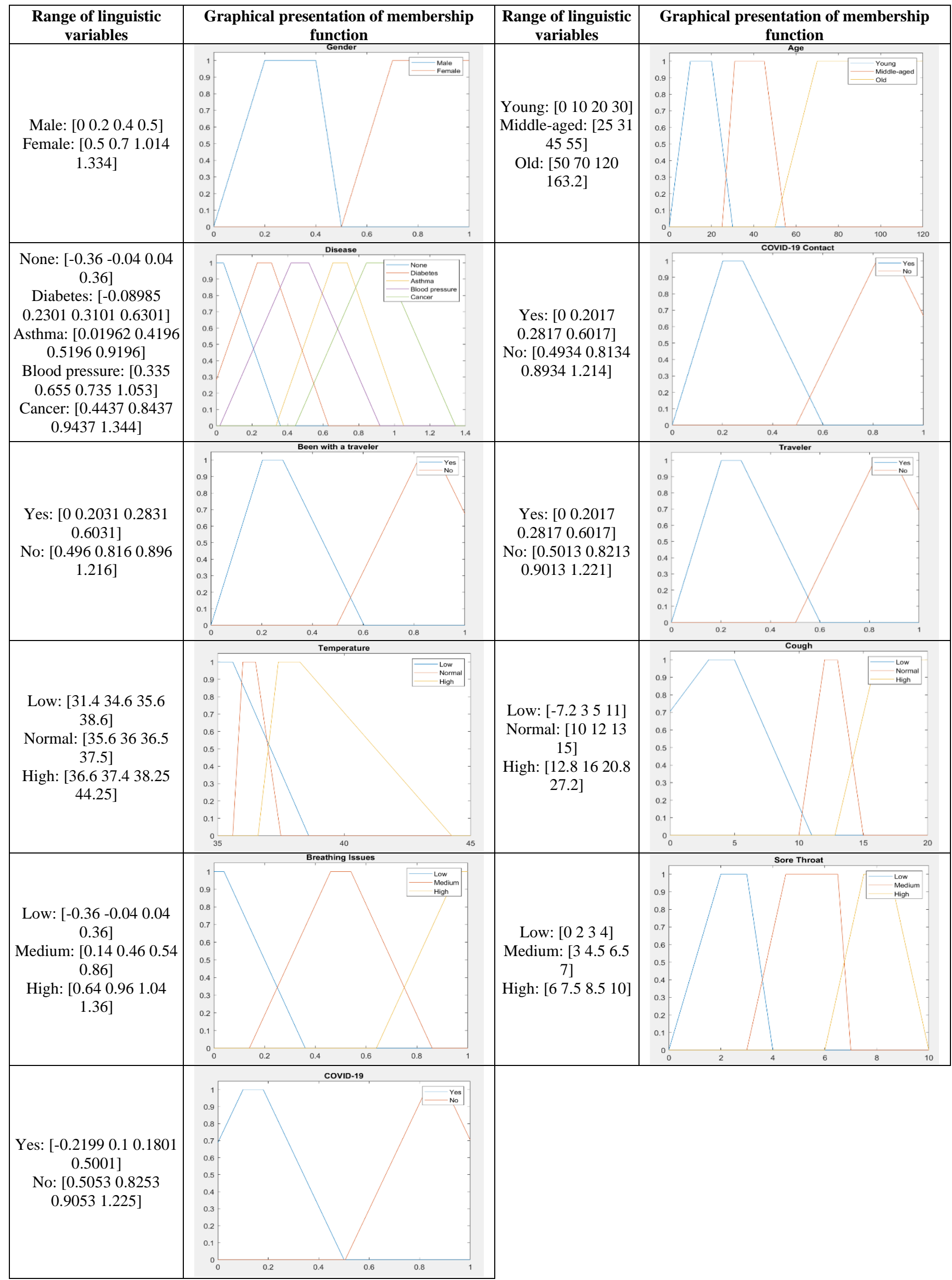




\subsubsection{Rules of the proposed model}

The system generates 49 rules for the proposed model, some of them are listed below.

1. If (Gender is Female) and (Age is Young) and (Diseases is None) and (Contact is Yes) and (WithTraveler is No) and (Travelled is No) and (Temperature is Normal) and (Cough is Low) and (Breathing_issue is Low) and (Sorethroat is Low) THEN (COVID-19 is No)

2. If (Gender is Female) and (Age is Young) and (Diseases is None) and (Contact is Yes) and (WithTraveler is Yes) and (Travelled is No) and (Temperature is Normal) and (Cough is Normal) and (Breathing_issue is Medium) and (Sorethroat is High) THEN (COVID-19 is Yes)

3. If (Gender is Female) and (Age is Young) and (Diseases is None) and (Contact is No) and (WithTraveler is No) and (Travelled is Yes) and (Temperature is Normal) and (Cough is Low) and (Breathing_issue is Low) and (Sorethroat is Low) THEN (COVID-19 is No)

4. If (Gender is Female) and (Age is Young) and (Diseases is None) and (Contact is Yes) and (WithTraveler is No) and (Travelled is Yes) and (Temperature is Normal) and (Cough is Low) and (Breathing_issue is Low) and (Sorethroat is Low) THEN (COVID-19 is No)

5. If (Gender is Male) and (Age is Young) and (Diseases is Diabetes) and (Contact is No) and (WithTraveler is No) and (Travelled is No) and (Temperature is Normal) and (Cough is Low) and (Breathing_issue is Low) and (Sorethroat is Low) THEN (COVID-19 is No)

6. If (Gender is Female) and (Age is Middle-aged) and (Diseases is None) and (Contact is Yes) and (WithTraveler is No) and (Travelled is No) and (Temperature is Normal) and
(Cough is Low) and (Breathing_issue is Low) and (Sorethroat is Low) THEN (COVID-19 is No)

7. If (Gender is Female) and (Age is Young) and (Diseases is None) and (Contact is Yes) and (WithTraveler is No) and (Travelled is No) and (Temperature is High) and (Cough is Low) and (Breathing_issue is Medium) and (Sorethroat is High) THEN (COVID-19 is Yes)

8. If (Gender is Male) and (Age is Young) and (Diseases is None) and (Contact is No) and (WithTraveler is No) and (Travelled is No) and (Temperature is Normal) and (Cough is Low) and (Breathing_issue is Low) and (Sorethroat is Low) THEN (COVID-19 is No)

3.2.6 Inferencing (two examples COVID-19 negative and positive)

The following example demonstrates a negative COVID-19 case using the bellow input and its corresponding linguistic variables.

Input.

[0.613;30.78;0.1087;0.2913;0.09914;0.6336;36.78;3.739;0.1 $522 ; 2.478]$

Linguistic variables. Gender: Female, Age: middle-aged, Diseases: None, Contact: yes, with traveler: yes, Traveler: no, Temperature: normal, Cough: low, Breathing_issue: low, Sore throat pain: low -> COVID-19: No.

Activated rule. (Gender is Female) and (Age is Middle-aged) and (Diseases is None) and (Contact is Yes) and (WithTraveler is Yes) and (Travelled is No) and (Temperature is Normal) and (Cough is Low) and (Breathing_issue is Low) and (Sorethroat is Low) then (COVID-19 is No).

Figure 3 presents the output for the previous input.

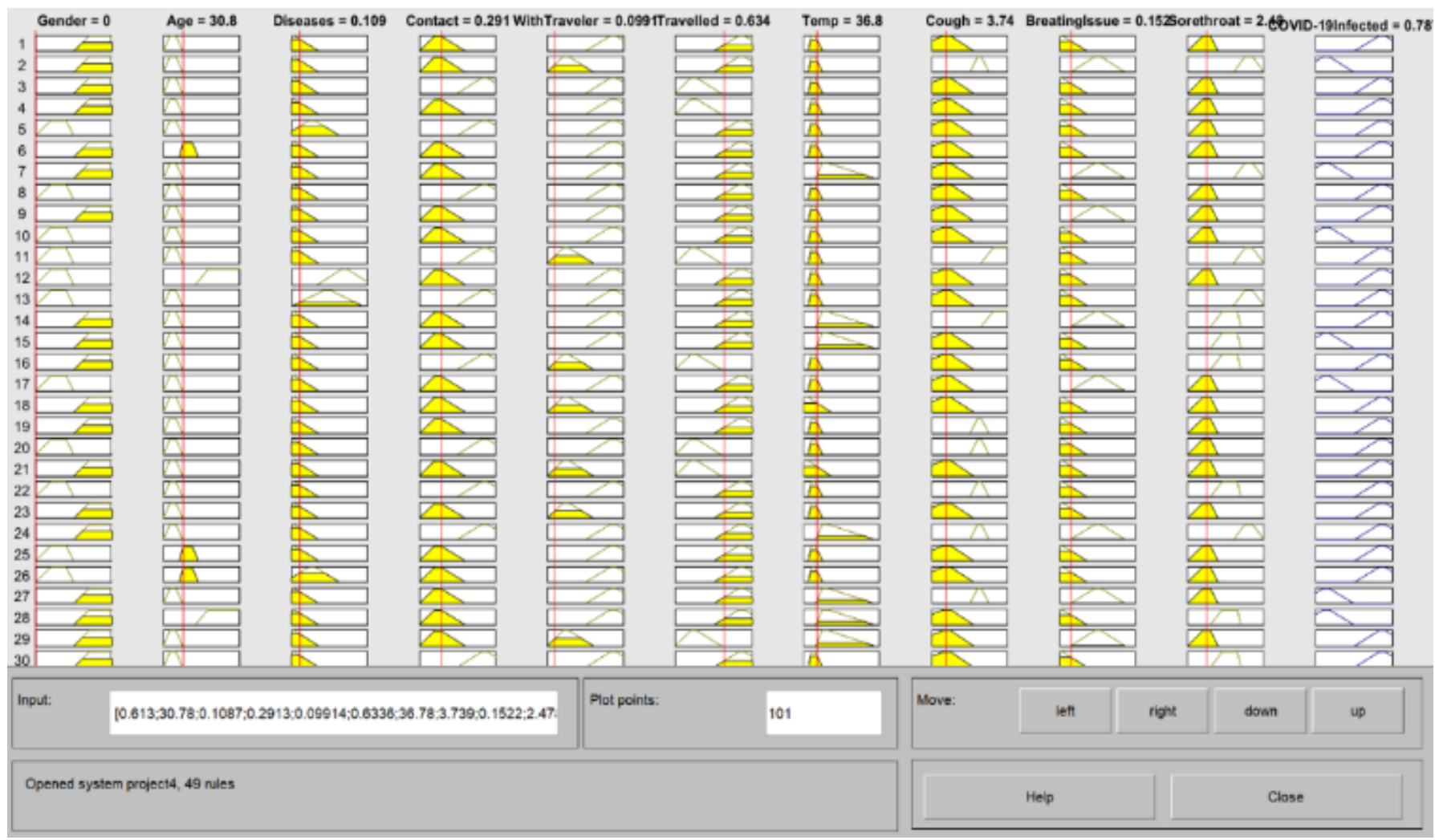

Figure 3. Rule inferencing COVID-19 negative 


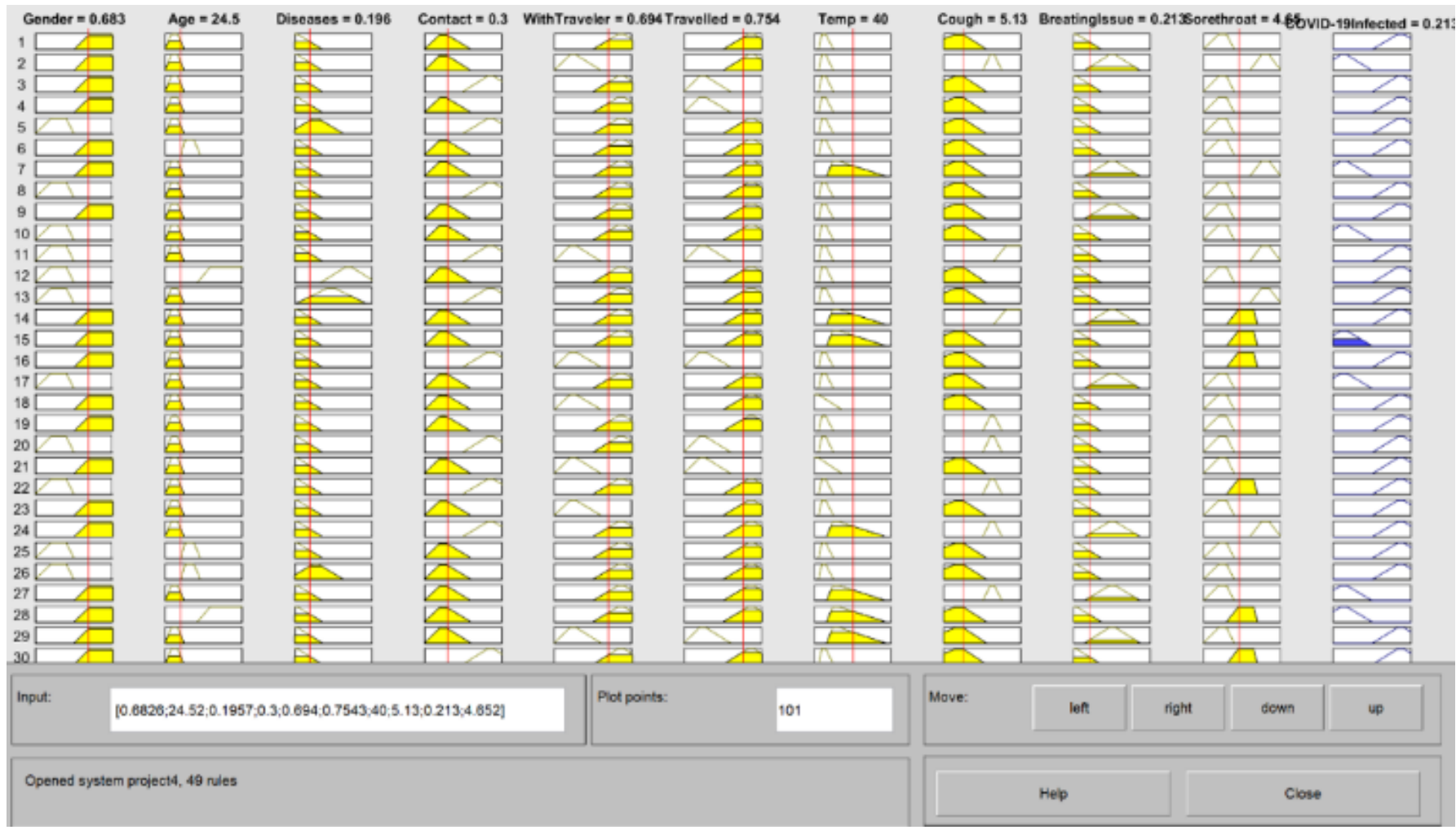

Figure 4. Rule inferencing COVID-19 positive

The following example demonstrates a positive COVID-19 case using the bellow input and its corresponding linguistic variables.

Input.

[0.6826;24.52;0.1957;0.3;0.694;0.7543;40;5.13;0.213;4.652]

linguistic variables. Gender: Female, Age: young, Diseases: None, Contact: yes, with traveler: no, Traveler: no, Temperature: high, Cough: low, Breathing_issue: low, Sore throat pain: medium -> COVID-19: Yes.

Activated rule. If (Gender is Female) and (Age is Young) and (Diseases is None) and (Contact is Yes) and (WithTraveler is No) and (Travelled is No) and (Temperature is High) and (Cough is Low) and (Breathing_issue is Low) and (Sorethroat is Medium) then (COVID-19 is Yes).

Figure 4 above presents the output for the previous input.

\section{RESULT AND DISCUSSION}

From the created IF-THEN rules, we can conclude that there is a relationship between gender, breathing issues, sore throat pain level, contact with a COVID-19 patient and being infected with COVID-19. The rules imply that if the gender was female, breathing issues or sore throat pain level were medium or high, contact with a COVID-19 patient is yes, then there is a high chance that the person is COVID-19 infected. According to the rules, $31.25 \%$ of females and $23.53 \%$ of males were COVID-19 positive, $38.71 \%$ of COVID-19 contact were COVID-19 positive, $54.55 \%$ of people with medium breathing issues were COVID-19 positive, and $55.56 \%$ of people with high sore throat pain were COVID-19 positive.

To evaluate the model performance, the following measures were used. Eq. (1) represents the accuracy formula, Eq. (2) represents the precision formula, Eq. (3) represents the sensitivity formula, Eq. (4) represents the specificity formula, and Eq. (5) represents the F1-score formula.

$$
\begin{gathered}
\text { Accuracy }=\frac{T N+T P}{T N+T P+F N+F P} \\
\text { Precision }=\frac{T P}{T P+F P} \\
\text { Sensitivity }(\text { Recall })=\frac{T P}{T P+F N} \\
\text { Specificity }=\frac{T N}{T N+F P} \\
F 1-\text { Score }=\frac{2 * \text { Precision } * \text { Recall }}{\text { Precision }+ \text { Recall }}
\end{gathered}
$$

The model gave the following result, an accuracy of $88.78 \%$, precision of $72.22 \%$, sensitivity of $68.42 \%$, specificity of $93.67 \%$, and an f1-score of $69.28 \%$. The accuracy of the model depends on the dataset quality. A fair and balanced dataset can improve the accuracy of the model further which makes it appropriate for the practical applications.

\section{COMPARISON OF THE PROPOSED MODEL WITH ANOTHER STUDY}

The study [27] had used MATLAB to simulate the model. Eleven types of fuzzy variables were used: cough, fever, age, travel history, diabetes, breathing problem, flu, hearing problem, smell losses, sore throat, and body aches to make predictions about coronavirus. The defuzzification part of the model takes input as fuzzy sets and matches membership 
degrees to generates crisp. A centroid form of De-Fuzzifier is used in the proposed model. If the patient has a fever and cough, the likelihood of coronavirus infection appears to be positive; similarly, if the patient has a fever greater than 38 and a dry cough, and the patient has trouble breathing and a travel history to infected countries, the result indicates a positive probability of coronavirus infection. Within the range of $0-1$, the model predicts 0.837 coronaviruses. In the experiment, Diabetes type I was considered not diabetes type II [28]. On the other hand, this paper uses MATLAB to simulate the model where the membership functions are automatically formed with respect to the given dataset values which make the proposed scheme dynamic compared to the studies [29, 30] where fixed values for the fuzzy membership functions were used. Ten different types of fuzzy variables are used for gender, age, temperature, cough, diseases (Asthma, diabetes, cancer, blood pressure), travel history, contact with a raveled person, contact with an infected person, breathing difficulty, and sore throat pain level to make predictions about coronavirus. If the patient is female, she has a high temperature, has contact with an infected person, and she suffers from medium difficulty breathing the likelihood of coronavirus infection appears to be positive; similarly, if the patient is male, he has a high sore throat, has contact with an infected person the likelihood of coronavirus infection appears to be positive. Within the range of $0-1$, our model predicts 0.787 coronavirus cases. It is apparent that the results obtained by proposed model are more accurate for the following reasons. Firstly, the other study considered only having or not having the symptom in which result is yes or no while this study considered the symptoms in ranges and only binary questions were considered as yes or no. Secondly, our study was based on a dataset while the other study was not utilizing a dataset, and finally, for the number of rules, the other study had only 15 rules without considering all the inputs and their ranges which we believe can oversimplify the model. However, our study had 49 rules considering all inputs and their possible ranges.

\section{CONCLUSIONS}

A rule-based fuzzy inference system was developed using survey data collected in Saudi Arabia to help in COVID-19 diagnosing. The system has 49 if-then rules that contain linguistic variables describing COVID-19 different symptoms along with other factors that are associated with the disease. The proposed study was compared with another study and the differences were outlined. The main limitation of the proposed study is that we used survey dataset, which is imbalanced and small sized, we tried to get reliable data from a hospital, but the data was incomplete. For future work, it is recommended to consider more COVID-19 symptoms and validating the methodology using real patients' data. Moreover, writing the proposed system in Python programming language using the inbuilt fuzzy system library to enable deploying the system in a website to make it available for a wide range of users.

\section{REFERENCES}

[1] Li, X.W., Geng, M.M., Peng, Y.Z., Meng, L.S., Lu, S.M. (2020). Molecular immune pathogenesis and diagnosis of COVID-19. Journal of Pharmaceutical Analysis, 10(2): 102-108. https://doi.org/10.1016/j.jpha.2020.03.001
[2] COVID-19: Who's at higher risk of serious symptoms? Mayo Clinic. https://www.mayoclinic.org/coronaviruswho-is-at-risk/art-20483301, accessed on Nov. 26, 2020.

[3] Hafeez, A., Ahmad, S., Siddqui, S., Ahmad, M., Mishra, S. (2020). A review of COVID-19 (Coronavirus Disease2019) diagnosis, treatments and prevention. Eurasian Journal of Medicine and Oncology, 4(2): 116-125. https://doi.org/10.14744/ejmo.2020.90853

[4] Naqvi, R.A., Mushtaq, M.F., Mian, N.A., Khan, M.A., Atta-ur-Rahman, Yousaf, M.A., Umair, M., Majeed, R. (2021). Coronavirus: A "mild" virus turned deadly infection. Computers, Materials \& Continua, 67(2): 2631-2646. https://doi.org/10.32604/cmc.2021.012167

[5] Dhiman, N., Sharma, M.K. (2020). Fuzzy logic inference system for identification and prevention of Coronavirus (COVID-19). International Journal of Innovative Technology and Exploring Engineering, 9(6): 1575-1580. https://doi.org/10.35940/ijitee.F4642.049620

[6] Fatima, S.A., Saleem, M., Asif, M., Hussain, N., Balouch, A., Rustam, I. (2020). IoT enabled Smart Monitoring of Coronavirus empowered with Fuzzy Inference System. https://www.researchgate.net/publication/339376183.

[7] Ahamad, M.K., Bharti, A.K. (2021). Prevention from COVID-19 in India: Fuzzy logic approach. 2021 International Conference on Advance Computing and Innovative Technologies in Engineering (ICACITE), pp. 421-426. https://doi.org/10.1109/ICACITE51222.2021.9404575.

[8] Khan, T.A., Abbas, S., Ditta, A. et al. (2020). Iomt-based smart monitoring hierarchical fuzzy inference system for diagnosis of COVID-19. Computers, Materials \& Continua, 65(3): 2591-2605. https://doi.org/10.32604/cmc.2020.011892

[9] Shaban, W.M., Rabie, A.H., Saleh, A.I., Abo-Elsoud, M.A. (2021). Detecting COVID-19 patients based on fuzzy inference engine and Deep Neural Network. Applied Soft Computing, 99: 106906. https://doi.org/10.1016/j.asoc.2020.106906

[10] Shatnawi, M., Shatnawi, A., Alshara, Z., Husari, G. (2021). Symptoms-based fuzzy-logic approach for COVID-19 diagnosis. International Journal of Advanced Computer Science and Applications, 12(4): 444-452.

[11] Zagrouba, R., Khan, M.A., Choudhry, M.A.S., et al. (2021). Modelling and simulation of COVID-19 outbreak prediction using supervised machine learning. Computers, Materials \& Continua, 66(3): 2397-2407. https://doi.org/10.32604/cmc.2021.014042

[12] Rahman, A., Sultan, K., Naseer, I., et al. (2021). Supervised machine learning-based prediction of COVID-19. Computers, Materials \& Continua, 69(1): 21-34. https://doi.org/10.32604/cmc.2021.013453

[13] Alotaibi, S.M., Rahman, A., Basheer, M.I., Khan, M.A. (2021). Ensemble machine learning based identification of pediatric epilepsy. Computers, Materials \& Continua, 68(1): 149-165 https://doi.org/10.32604/cmc.2021.015976

[14] Rahman, A., Salam, M.H., Jamil, S. (2013). Virtual clinic: A telemedicine proposal for remote areas of Pakistan. 3rd World Congress on Information and Communication Technologies (WICT'13), pp. 46-50. https://doi.org/10.1109/WICT.2013.7113107

[15] Rahman, A. (2020). GRBF-NN based ambient aware realtime adaptive communication in DVB-S2. Journal of Ambient Intelligence and Humanized Computing, 
2020(12): 1-11. https://doi.org/10.1007/s12652-02002174-w

[16] Rahman, A., Qureshi, I.M., Malik, A.N., Naseem, M.T. (2016). QoS and rate enhancement in DVB-S2 using Fuzzy Rule Base System. Journal of Intelligent \& Fuzzy Systems $\quad$ (JIFS), $\quad 30(2)$ : $\quad$ 801-810. https://doi.org/10.3233/IFS-151802

[17] Rahman, A., Dash, S., Luhach, A.K., Chilamkurti, N., Baek, S., Nam, Y. (2019). A neuro-fuzzy approach for user behavior classification and prediction. Journal of Cloud Computing, $8(17)$. https://doi.org/10.1186/s13677-019-0144-9

[18] Zaman, G., Mahdin, H., Hussain, K., Atta-Ur-Rahman, Abawajy, J., Mostafa, S.A. (2021). An ontological framework for information extraction from diverse scientific sources. IEEE Access, 9: 42111-42124. https://doi.org/10.1109/ACCESS.2021.3063181

[19] Rahman, A., Sultan, K., Aldhafferi, N., Alqahtani, A. (2018). Education data mining for enhanced teaching and learning. Journal of Theoretical and Applied Information Technology, 96(14): 4417-4427.

[20] Rahman, A., Dash, S. (2017). Data mining for students' trends analysis using Apriori Algorithm. International Journal Control Theory and Applications, 10(18): 107115.

[21] Fuzzy Sets and Pattern Recognition. https://www.cs.princeton.edu/courses/archive/fall07/cos 436/HIDDEN/Knapp/fuzzy004.htm, accessed on Apr. 30, 2021

[22] Zadeh, L.A. (2009). Toward extended fuzzy logic-A first step. Fuzzy Sets and Systems, 160(21): 3175-3181. https://doi.org/10.1016/j.fss.2009.04.009

[23] Sabahi, F., Akbarzadeh-T, M.R. (2013). A qualified description of extended fuzzy logic. Information
Sciences,

244:

$60-74$.

https://doi.org/10.1016/j.ins.2013.03.020

[24] Rahman, A., Sultan, K., Naseer, I., et al. (2021). Supervised machine learning-based prediction of COVID-19. Computers, Materials \& Continua, 69(1): 21-34. https://doi.org/10.32604/cmc.2021.013453

[25] Rahman, A. (2019). Optimum information embedding in digital watermarking. Journal of Intelligent and Fuzzy Systems, 37(1): 553-564. https://doi.org/10.3233/JIFS162405

[26] Rahman, A., Qureshi, I.M., Malik, A.N., Naseem, M.T. (2014). Dynamic resource allocation for OFDM systems using DE and fuzzy rule base system. Journal of Intelligent \& Fuzzy Systems (JIFS), 26(4): 2035-2046. https://doi.org/10.3233/IFS-130880

[27] Painuli, D., Mishra, D., Bhardwaj, S., Aggarwal, M. (2020). Fuzzy rule based system to predict COVID19-A deadly virus. International Journal of Management and Humanities, 4(8): 78-82. https://doi.org/10.35940/ijmh.H0781.044820

[28] Rehman, A., Athar, A., Khan, M.A., et al. (2020). Modelling, simulation, and optimization of diabetes type II prediction using deep extreme learning machine. Journal of Ambient Intelligence and Smart Environments, 12(2): 125-138. https://doi.org/10.3233/AIS-200554

[29] Naseem, M.T., Qureshi, I.M., Rahman, A., et al. (2020). Robust and fragile watermarking for medical images using redundant residue number system and chaos. Neural Network World, 30(3): 177-192. https://doi.org/10.14311/nnw.2020.30.013

[30] Rahman, A. (2019). Memetic computing based numerical solution to Troesch problem. Journal of Intelligent and Fuzzy Systems, 37(1):1545-1554. https://doi.org/10.3233/JIFS-18579 